\title{
Micro- and nano-tomography at the DIAMOND beamline I13L imaging and coherence
}

C. Rau, A. Bodey, M. Storm, S. Cipiccia, S. Marathe, et al.

C. Rau, A. Bodey, M. Storm, S. Cipiccia, S. Marathe, M.-C. Zdora, I. Zanette, U. Wagner, D. Batey, X. Shi, "Micro- and nano-tomography at the DIAMOND beamline I13L imaging and coherence," Proc. SPIE 10391, Developments in X-Ray Tomography XI, 103910T (11 October 2017); doi: 10.1117/12.2274514

SPIE Event: SPIE Optical Engineering + Applications, 2017, San Diego, California, United States 


\title{
Micro- and Nano-Tomography at the DIAMOND beamline I13L Imaging and Coherence
}

\author{
C. Rau$^{* 1,2,3}$, A. Bodey ${ }^{1}$, M. Storm ${ }^{1}$, S. Cipiccia ${ }^{1}$, S. Marathe ${ }^{1}$, M.-C. Zdora ${ }^{1,4}$, I. Zanette ${ }^{1}$ \\ U. Wagner ${ }^{1}$, D. Batey ${ }^{1}$, X. Shi ${ }^{1}$. \\ ${ }^{1}$ Diamond Light Source Ltd., Harwell Science and Innovation Campus, Didcot, OX 11 0DE, UK. \\ ${ }^{2}$ University of Manchester, School of Materials Grosvenor St., Manchester, M1 7HS, UK. \\ ${ }^{3}$ Northwestern University, 303 E. Chicago Avenue, Chicago, IL 60611-3008, USA. \\ ${ }^{4}$ Department of Physics \& Astronomy, University College London, London WC1E 6BT, UK
}

\begin{abstract}
The Diamond Beamline I13L is dedicated to imaging on the micro- and nano-lengthsale, operating in the energy range between 6 and $30 \mathrm{keV}$. For this purpose two independently operating branchlines and endstations have been built. The imaging branch is fully operational for micro-tomography and in-line phase contrast imaging with micrometre resolution. Grating interferometry is currently implemented, adding the capability of measuring phase and small-angle information. For tomography with increased resolution a full-field microscope providing $50 \mathrm{~nm}$ spatial resolution with a field of view of $100 \mu \mathrm{m}$ is being tested. The instrument provides a large working distance between optics and sample to adapt a wide range of customised sample environments. On the coherence branch coherent diffraction imaging techniques such as ptychography, coherent X-ray diffraction (CXRD) are currently developed for three dimensional imaging with the highest resolution.

The imaging branch is operated in collaboration with Manchester University, called therefore the Diamond-Manchester Branchline. The scientific applications cover a large area including bio-medicine, materials science, chemistry geology and more. The present paper provides an overview about the current status of the beamline and the science addressed.
\end{abstract}

Keywords: tomography, synchrotron radiation, nano-imaging, phase contrast, X-ray microscopy

\section{INTRODUCTION}

The unique properties of synchrotron radiation enable new science exploring advanced X-ray imaging methods. The partial coherence of the radiation enables in-line phase contrast imaging, suitable for weakly absorbing structures and many applications for bio-medical research. The high photon flux is used for rapid imaging and the energy tunability can provide chemical information. Imaging with highest spatial resolution is accessible by advanced methods relying on highly performing X-ray optics.

The Diamond beamline I13L is operational since 2011 and provides micro- and nano-imaging and tomography[1]. Dedicated sample environments are developed in junction with collaborators, providing a large variety of scientific applications. Some methods such as micro-tomography operate in standard user operation mode, while some other instrumentation is currently under development.

\section{THE BEAMLINE}

The source of the beamline is located in one of the long straight sections of the Diamond electron storage ring. This long straight section has been divided into two shorter parts, reducing the vertical electron size in each sub-section and thus allowing smaller insertion device gaps. In combination with a modified magnetic layout of the undulator, the brilliance for each source is increased by one order of magnitude compared to the original layout. This electron-optical layout of the storage ring is called 'mini-beta', referring to the reduced vertical electron size. In each sub-section of this modified

Developments in X-Ray Tomography XI, edited by Bert Müller, Ge Wang, Proc. of SPIE Vol. 10391,

103910T - (c) The Authors. Published under a Creative Commons Attribution CC-BY 3.0 License · doi: 10.1117/12.2274514 
straight section an undulator is placed. They can be operated individually and are canted to each other. More details about the optical layout can be found elsewhere [1,2]. More than $200 \mathrm{~m}$ from the source two experimental stations are operating independently. Together they cover several orders of magnitude in terms of spatial resolution, starting at the micro -lengthscale and finally aiming for sub-ten nanometer resolution.

The imaging branchline is mainly dedicated to real-space imaging techniques. Because of the close collaboration with Manchester University and their dedicated financial engagement and commitment for staffing, this branch is also called the 'Diamond Manchester imaging branchline'. The collaboration develops unique sample environments and experimental rigs, in sight to make them available for the user community. Most scientists from the Manchester collaboration and other Universities make use of in-line phase contrast imaging as a versatile tool for their studies. The resolution is in the micron range. A second instrument, full-field transmission microscope is currently developed, providing about 50-100 $\mathrm{nm}$ over a large field of view.

The coherence branchline applies imaging techniques in reciprocal space with currently $30 \mathrm{~nm}$ spatial resolution and better in the near future. The main techniques used are ptychography and Bragg-coherent diffraction imaging.

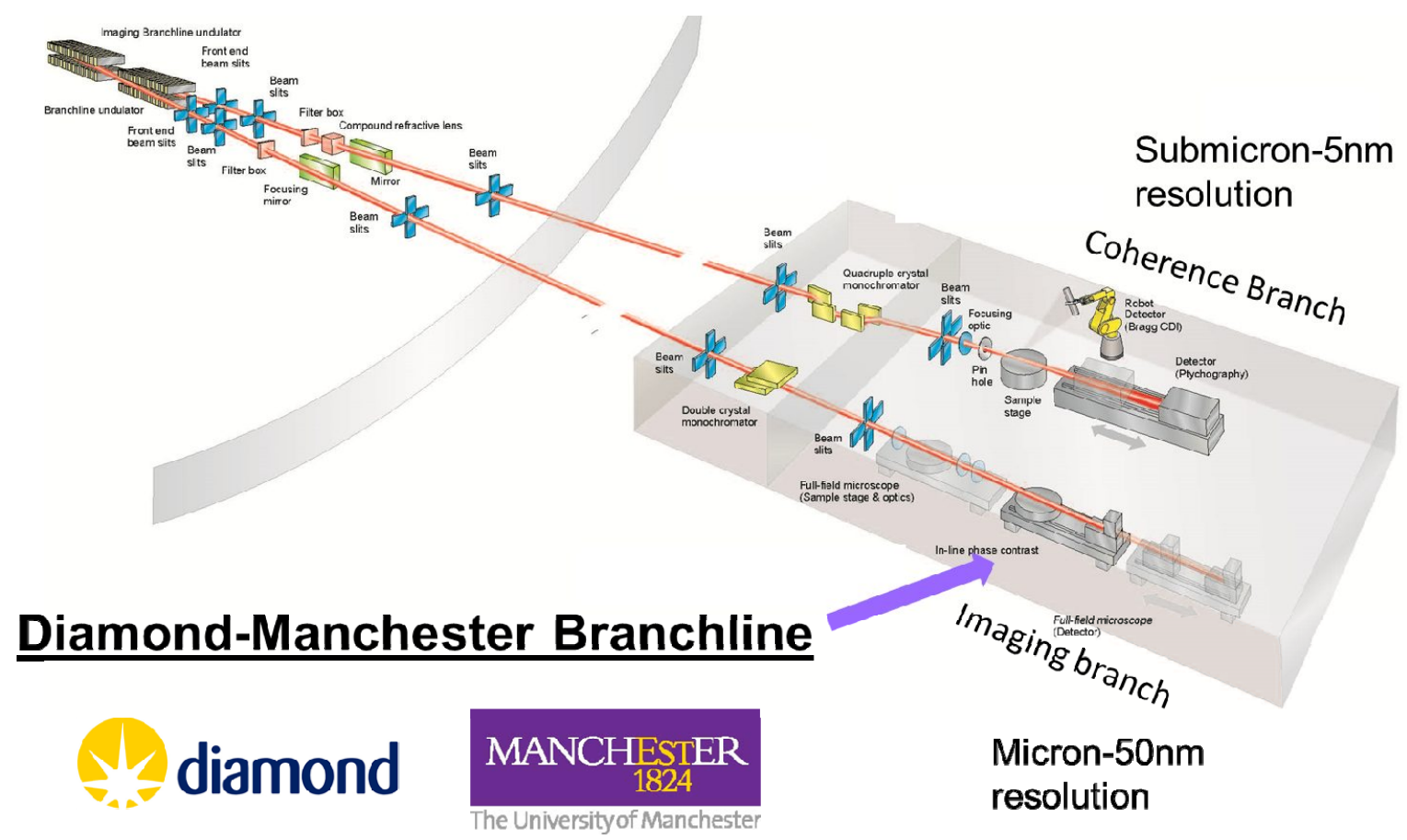

Figure 1: Schematic overview of the experimental stations at the Diamond beamline I13L

\subsection{The Diamond-Manchester Imaging branchline}

\subsubsection{In-line phase contrast imaging}

The in-line phase contrast setup consists of a sample stage and a detector section. The distance between both can be increased up to two meters to vary the contrast. The sample is aligned in the beam with a stack of height and tilt stages [3]. For tomography the air bearing stage has a horizontal run-out of less than $50 \mathrm{~nm}$, a wobble of $0.1^{\circ}$ (PI Micos UPR270) and is used for micro- and nano-tomography. The individual samples are aligned to the rotation center with stick-slip nano-positioning actuators (SmarAct). The minimal translation increment of the stage is $4 \mathrm{~nm}$ and the repeatability is about $30 \mathrm{~nm}$. The stack of stages can be horizontally moved out of the beam for a hexapod stage. This stage supports experiments with dedicated stress and torsion rigs and tomography with customized sample environments (see paragraph 3: sample environments and science). Two detector systems can be used during the same experiment by 
swapping them in and out with a horizontal translation stage. Each detector system works with a scintillation screen which transforms the X-rays into visible light, the visible light image on the scintillation screen is then projected trough a visible light microscope on the chip of the CCD or CMoS camera. The pairs of scintillation screen and microscope objective lens can be changed with a revolver mechanism for adapting different magnifications. The field of view of the interchangeable optics ranges from less than $1 \times 1 \mathrm{~mm}^{2}$ to $10 \times 15 \mathrm{~mm}^{2}$ [4]. One detector system is equipped with a PCO4000 CCD detector for longer exposure times and a large field of view and a PCOedge CMoS detector. The PCOedge CMoS detector is most frequently used and it can record up to 100 images per second. Typical tomogram recording times are ranging from seconds to a few minutes. A third detector system based on the PCODimax is dedicated to rapid imaging with a rate of more than 1200 frames per second in full-frame mode and $40 \mathrm{~ms}$ minimal exposure time. The updated list for available detectors and detector system optics are listed on the I13 webpage [4]. Recent efforts in the development of the beamline have been focused on improving usability and reliability, especially for data recording.

\subsubsection{Grating interferometry and speckle imaging}

In-line phase contrast explores the coherence of X-ray for enhancing the structure of weakly absorbing materials. More information, such as the phase shift induced by material or its small-angle properties can be studied with grating interferometry and speckle imaging, which is currently tested at I13L[5,6]. The refractive index of the material can be measured. Even more interesting is the fact that in the operational energy range of the beamline the phase signal is two orders more sensitive than the one relating to absorption. This is very helpful for segmenting samples or measuring small variations -for example for bone density- with high precision. An example for enhanced data segmentation can be found in [5], more information is provided by the article of S. Marathe in the present proceedings. The small angle signal enables distinguishing matter on the nano-scale, hierarchical structures or eventually the formation of separated phases in a homogenous environment. The applications range from bio-medical examples (bone-cartilage), energy materials to materials science.

\subsubsection{Full-field microscope (TXM)}

Details of the pilot instrument TXM are described elsewhere [7](see figure 2), the essential characteristics are summarized in this section. For the optical layout of the instrument the working distance between sample and objective lens is larger than $50 \mathrm{~mm}$ to allow for space of dedicated sample environments. A field of view close to $100 \mu \mathrm{m}$ provides ease for sample preparation and corresponds to the microscope's depth of focus. Tomography is applicable over the entire field of view for the sample. The large field of view is enabled by a specific microscope condenser optic, called 'beam shaper'. This device consists of an array of diffractive optics, each segment focuses on the sample plane.

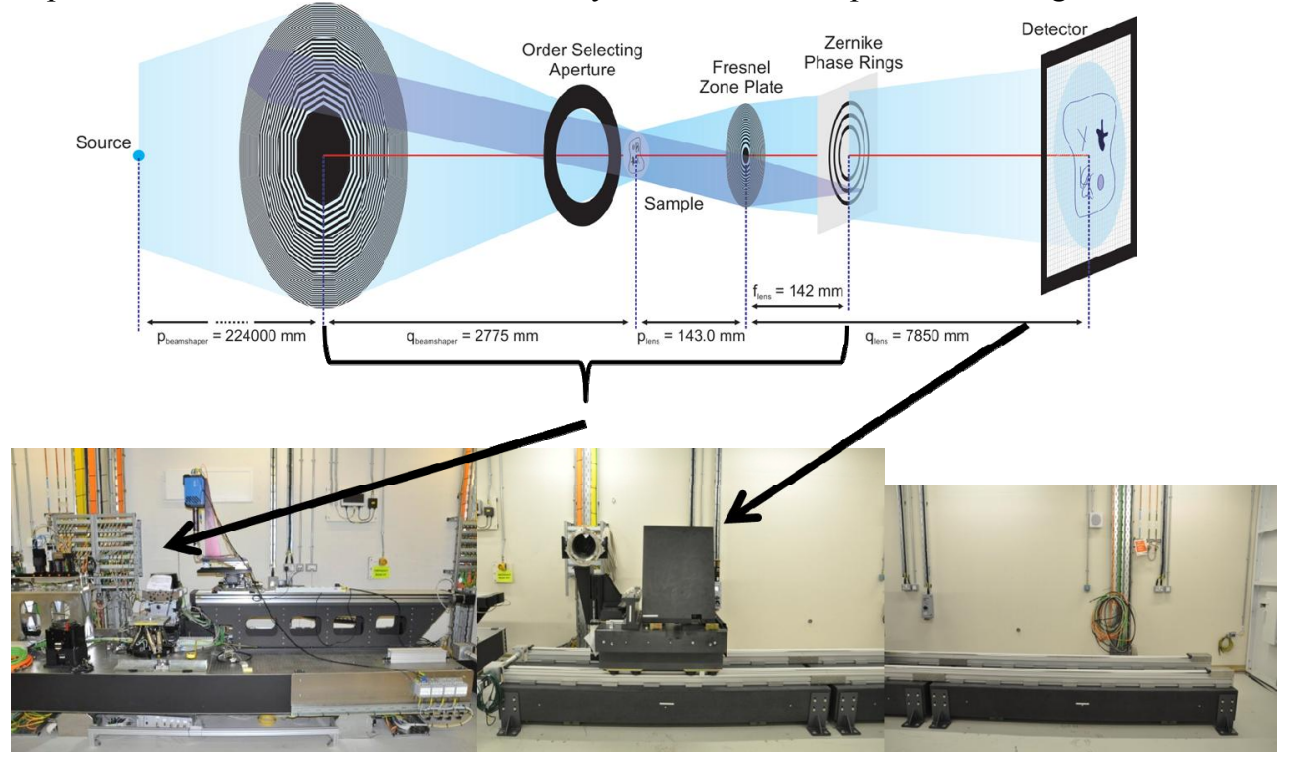

Figure 2: Scheme of optical layout of TXM (top), and photo of pilot instrument (bottom) [7]. 
The instrument provides imaging in Zernike phase contrast mode. The early version of the instrument operated with monochromatic beam of a Si (111) monochromator and the PCO4000 detector system of the micro-tomography setup. Two sets of X-ray optics for 8.3 and $11.5 \mathrm{keV}$ (collaboration C. David, PSI) are currently used. Most recently more broadband radiation from a multilayer monochromator (MLM) has been successfully tested and a more efficient, medium-resolution detector is used. Three different multilayer systems $\left(\mathrm{Mo}-\mathrm{B}_{4} \mathrm{C} ; \mathrm{Ru}-\mathrm{B}_{4} \mathrm{C} ; \mathrm{V}-\mathrm{B}_{4} \mathrm{C}\right)$ of the monochromator cover the energy range of the beamline with energy bandwidth $\Delta \mathrm{E} / \mathrm{E}$ of $10^{-3}$ to $10^{-2}$. The flux is increased by about a factor of 20 compared to monochromatic beam. The Hamamatsu C12849-101U X-ray sCMOS detector has a $2048 \times 2048$ array with 6.5 micron pixel size and a $10 \mu \mathrm{m}$ thick Gadox scintillator. With the current version of the instrument typical exposure times are in the range of some hundred millisecond and full tomograms can be recorded within minutes.

\subsection{The coherence branchline}

The spatial resolution for imaging in direct space is either limited by the detector resolution (for in-line phase contrast) or the X-ray optics used (the outermost zone width of the zone plates of the transmission microscope). Techniques such as ptychography and Bragg-CDI operating in the far-field/reciprocal space overcome these limitations. Currently ptychography and Bragg-CDI are developed at the coherence branchline for user operation. The basic instrumentation is similar to the imaging branch in terms of sample and detector stages currently used $[8,9]$. For both experiments Ptychography and Bragg-CDI- different optics such as Fresnel-Zone plates (FZP) and Kirkpatrik-Baez (KB) mirrors are available for focusing the beam to spot sizes between $50 \mathrm{~nm}$ to $10 \mu \mathrm{m}$. The distance between sample and detector can be large: in the case of ptychography up to $18 \mathrm{~m}$ and for Bragg-CDI up to $8 \mathrm{~m}$ (with some limitations in the angular range for the latter). The variability in terms of spot size and detector-sample distance provides a zooming capability for the branchline, which is a unique feature for this type of techniques. The detectors for the branchline are based on the single photon counting MediPixIII chip and a pixel size of $55 \mu \mathrm{m}$. The EXCALIBUR detector has been developed in junction with STFC $[10,11]$, measures a $2 \mathrm{k} \times 1.5 \mathrm{k}$ pixel array, which can be read out with $100 \mathrm{~Hz}$ in continuous mode and $1 \mathrm{kHz}$ in burst mode. The detector is either held on a translation stage or for Bragg-CDI on a robot arm (see figure 3). The latter provides the necessary stability and drifts of few microns over the time scale of hours [8]. Today, ptychography can be performed with a rate faster than $10 \mathrm{~Hz}$ and a resolution better than $30 \mathrm{~nm}$, using specific scanning software originally developed for serial crystallography [12]. In the near future stages for the sample will be upgraded with piezo stages (ptychography), for Bragg-CDI the angular range will be increased. The ptychography and Bragg-CDI measurements can also be complemented with fluorescence information. Currently a single element Vortex X-ray Detector with XMap readout is used. The maximum detectable energy is $20 \mathrm{keV}$.

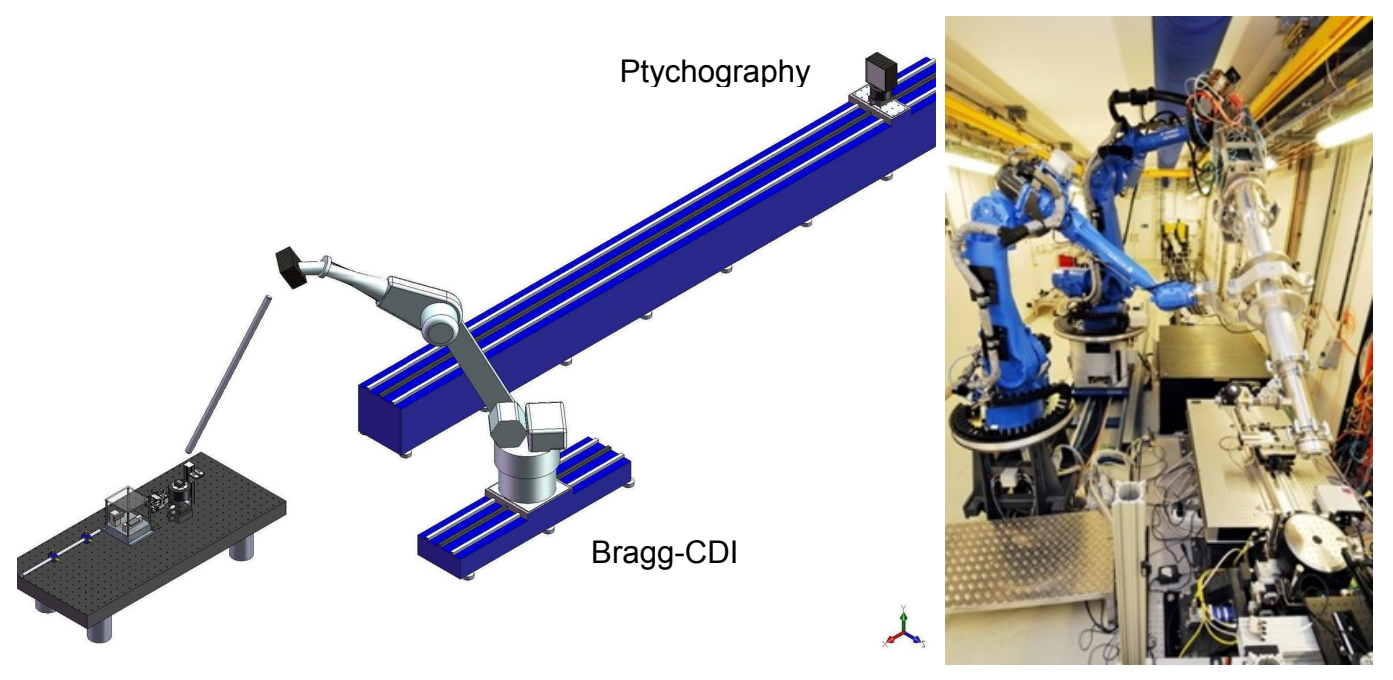

Figure 3: Layout scheme for experiments on I13 coherence branch (left) and photo of experiment and robot arms. 


\section{SAMPLE ENVIRONMENTS AND SCIENCE}

Most interesting are the developments of scientific applications with dedicated sample environments. Some examples are listed in the following.

\section{$\underline{3.1 \text { Nuclear graphite }}$}

Graphite is a moderator material for neutrons in nuclear reactors. Its long term stability is highly relevant for the industrial use in fission power stations. The micro-structure is studied under different conditions, such as heat and mechanical load. The rig and the sample environment used for the measurements are shown in figure 4. Pressure and torsion can be applied with an Open-Frame Rig (Deben; see figure 4 left). Two rotation stages, placed above and below the sample, (see figure 4, left blue part) can be operated indepently and the distance between both can be changed. Two infrared lamps focus from opposite directions onto the sample (figure 4 centre and right). Two encapsuled glass cylinder enclose and secure the radioactive material.

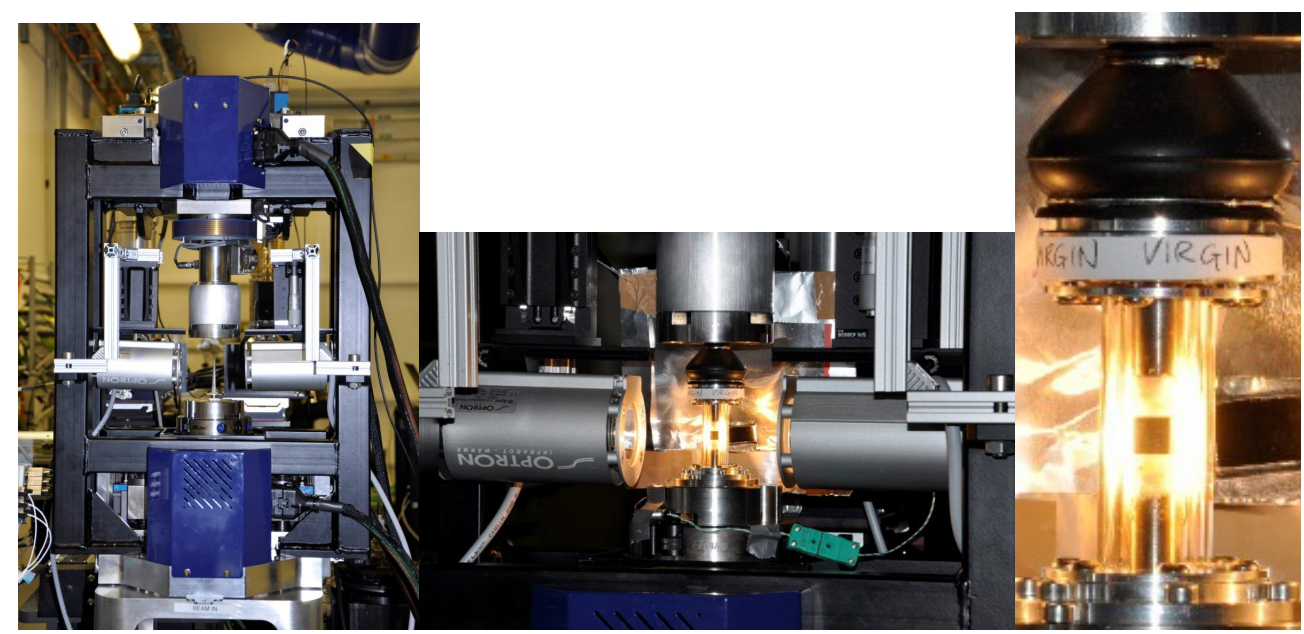

Figure 4: Sample environment for studying nuclear graphite.

Left: torsion rig with two independent rotation stages above and below the sample Centre and right: double glass enclosure for sample and surrounding heating units

First results of this study are shown in figure 5 [13]. The experiment was carried out with polychromatic 'pink-beam', taking 2001 projections with $75 \mathrm{~ms}$ exposure time. Using an effective pixel size of $2.6 \mu \mathrm{m}$ on the detector, the resolution is estimated to be around $6 \mu \mathrm{m}$. The measurements compare the micro-structure of unirradiated and irradiated graphite, both samples heated up to $300^{\circ} \mathrm{C}$ and exposed to a load of $450 \mathrm{~N}$. The significant structural difference between the two types of samples is obvious as shown in the reconstructed sections. Radiation and radiolytic oxidation cause a significant increase of the material's porosity and simultaneous compression of filler particles (see circle in figure 5). The results serve for theoretical models for predicting the change of the thermal expansion coefficient - and therefore the expansion behavior - for graphite rods during their lifetime.
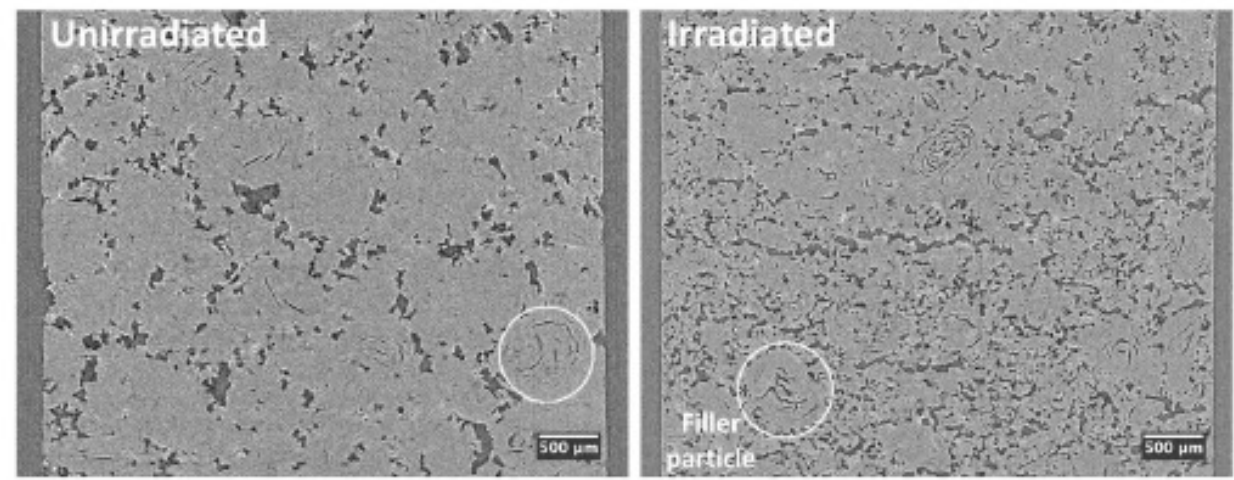

Figure 5: Section through reconstructed volume of unirradiated (left) and irradiated (right) nuclear graphite [13]. 


\subsection{Ice cream, $\mathrm{CO}_{2}$ storage and Batteries}

Some more examples for experiments with dedicated sample environment are listed in the following and details are provided in the corresponding publications. One original experiment is about ice cream. The taste of ice cream is significantly influenced by the microstructure of its components. A specific box has been designed for the experiment with the capabilities to go through several melting-freezing cycles to induce the typical changes which may occur [see figure 6 left) [14]. The results show the coarsening of both the air cells and ice crystals in ice cream, which finally result in a change in taste.

The first fast scans (some seconds per tomogram) at the beamline were carried out on the study of $\mathrm{CO}_{2}$ storage in brine and sandstone. For this experiment a dedicated system has been designed, consisting of a pumping system putting up a pressure of up to 100 bars and the container with the sandstone-brine- $\mathrm{CO}_{2}$ mix (setup and 'pink' beam see figure 6, right) [15-17]. With the setup it has been possible to learn about the connectivity between the different components, which is essential to understand the mechanism of storing $\mathrm{CO}_{2}$.

Battery materials require special enclosures for protecting the often very reactive composites such lithium from the outer atmosphere. The material has to be at the same time sufficiently transparent for X-rays. Initially kapton cells were used and are now replaced by more advanced designs using other cell materials[18, 19].
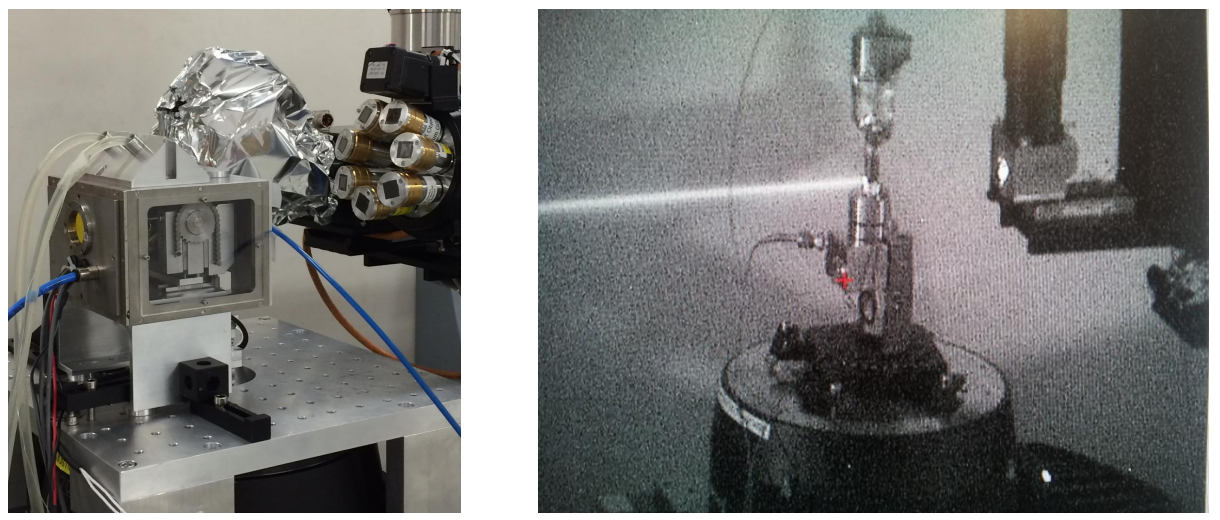

Figure 6: Sample environment for study of ice cream (left); storage of supercritical CO2 in brine and sandstone studied with high-pressure cell and 'pink' beam (right).

\section{$\underline{3.3 \text { Fukushima fallout particles }}$}

The Fukushima reactor accident in 2011 produced nuclear fallout. The particles found in the following provide valuable information for possible accident scenarios. The collected samples were sealed in double walled kapton containers (see figure 7, left) for the experiments on the I13 coherence branchline. The ptychography and fluorescence measurements permit to learn about the morphology and the chemical composition of the sample. While the ptychography data provides a $2 \mathrm{D}$ resolution of about $30 \mathrm{~nm}$, the spectroscopic data are in the micron range. Ideally, the ptychographic data may help improving the resolution of the spectroscopic information.

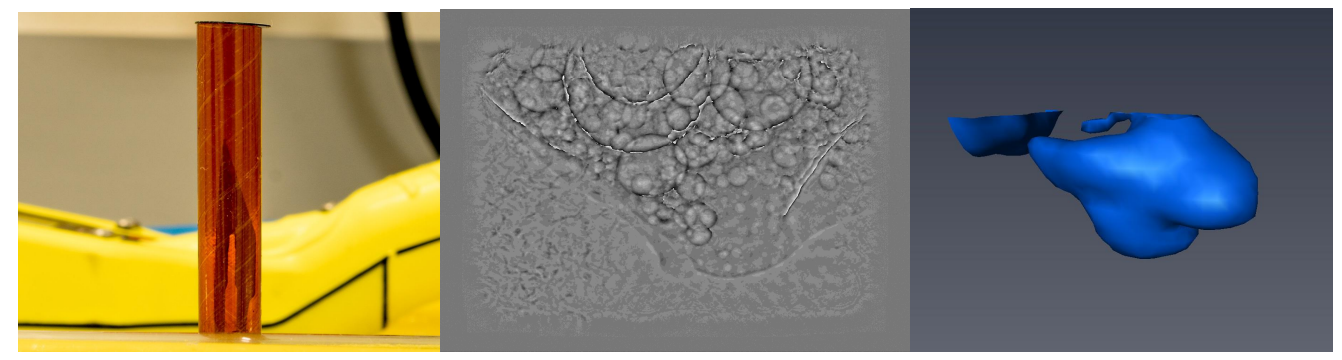

Figure 7: Sample environment and measurements of particles from Fukushima fallout

Left: Double wall container for radioactive samples for ptychographic measurements.

Centre: object phase reconstructed from a 2D ptychography scan.

Right: 3D Distribution of the manganese obtained from the fluorescence data. 


\subsection{Sample environments and Manchester collaboration}

For the future it is planned to provide a choice of different sample environments for user operation, to be booked online or requested jointlyin with the beamtime application. Many of these sample environments are purchased by the renewed Manchester collaboration. This collaboration ensured the completion and the proper staffing of the imaging branchline in the past and continues supporting the scientific program at $113 \mathrm{~L}$ now.

\section{I13-DATA BRANCHLINE}

The beamline supports the data analyses and the follow-up of experiments via a structure similar to the operation of the I13 branchlines. The data branchline is embedded within the Diamond User Administration System (UAS). Data analyses sessions can be scheduled similar to real experiments. The costs for users are covered, the schedule for the data analyses facility is ensured and the users are prioritized for the computer cluster usage (the schedule is preferentially during machine shutdown). A staff-supported office with several high performance visualization machines (details described in [20]) can host two independent science teams.

The activity ensures the follow up of data processing to publication level, helps user groups with limited experience in imaging and limited equipment available at their home institute and enhances the exchange of information between the beamline team, users and eventually other science teams present at the beamline. As the data can be processed more rapidly via the data branchline, this also reduces the load on the storage system for the raw imaging data..

\section{SUMMARY}

Micro- and nano-tomography is provided the Diamond Beamline I13L on two independently operating branchlines. The Diamond Manchester imaging branchline covers micron and submicron resolution with in-line phase contrast imaging, grating interferometry and full-field transmission microscopy. The coherence branch offers unique zooming capabilities for ptychography and Bragg-CDI with variable beamsize and sample-detector distance. The beamline currently enhances its capabilities for the most advanced techniques and incorporates dedicated sample environments, which will be available for a large user community.

The beamline's scientific program aims for multi-scale and in-operando research with applications in diverse areas such as biology, materials science, geology, energy materials and climato-paleantology.

\section{ACKNOWLEDGEMENTS}

The Diamond Manchester collaboration is acknowledged for financial and logistic support. The I13 team closely collaborates with the group of C. David (PSI) for X-ray optics. Joan Vila is acknowledged for his significant contributions for the imaging branch and initial work for the TXM. Kaz Wanelik and Andy Wilson provide excellent support for the beamline controls and data acquisition. The team thanks the colleagues from the Diamond I24 beamline (Owen Robin) for their significant help to improve ptychographic scanning at our beamline. Numerous collaborators are recognized for their contributions, namely the teams of Pierre Thibault, Ian Robinson, John Rodenburg and Marcus Newton for coherent imaging. The collaborators on the imaging branch are numerous, for the Manchester collaboration the director Neil Bourne, David Eastwood and also for past work the team of P. Lee are acknowledged.

\section{REFERENCES}

[1] C. Rau, U. Wagner, Z. Pesic et al., "Coherent imaging at the Diamond beamline I13," Physica Status Solidi (a), 208(11), 2522-2525 (2011). 
[2] B. Singh, R. Bartolini, R. Fielder et al., "Double Mini-beta-Y Plus Virtual Horizontal Focussing Optics for the Diamond Storage Ring," PAC09 Conf. Proc, 506, (2009).

[3] Z. Pešić, A. De Fanis, U. Wagner et al., "Experimental stations at I13 beamline at Diamond Light Source," Journal of Physics: Conference Series, 425, 182003 (2013).

[4] http://www.diamond.ac.uk/Beamlines/Materials/I13/DiamondManchester_Imaging Branchline/Facilities and equipment Imaging.html.

[5] M. C. Zdora, J. Vila-Comamala, G. Schulz et al., "X-ray phase microtomography with a single grating for highthroughput investigations of biological tissue," Biomedical Optics Express, 8(2), 1257-1270 (2017).

[6] M. C. Zdora, P. Thibault, T. Zhou et al., "X-ray Phase-Contrast Imaging and Metrology through Unified Modulated Pattern Analysis,” Physical Review Letters, 118(20), 203903 (2017).

[7] J. Vila-Comamala, J. Bosgra, D. S. Eastwood et al., "Transmission X-ray Microscopy at Diamond-Manchester I13 Imaging Branchline," XRM 2014: Proceedings of the 12th International Conference on X-Ray Microscopy, 1696, 020036 (2016).

[8] Z. Pešić, U. Wagner, and C. Rau, "Design of the experimental stage for coherent diffraction imaging."

[9] Z. Pešić, A. De Fanis, U. Wagner et al., "Experimental stations at I13 beamline at Diamond Light Source," Journal of Physics Conference Series. 425.

[10] N. Tartoni, G. Dennis, P. Gibbons et al., "Excalibur: a three million pixels photon counting area detector for coherent diffraction imaging based on the Medipix3 ASIC," 2012 Ieee Nuclear Science Symposium and Medical Imaging Conference Record, 530-533 (2012).

[11] J. Marchal, I. Horswell, B. Willis et al., "EXCALIBUR: a small-pixel photon counting area detector for coherent X-ray diffraction - Front-end design, fabrication and characterisation," 11th International Conference on Synchrotron Radiation Instrumentation, 425, 062003 (2013).

[12] D. A. Sherrell, A. J. Foster, L. Hudson et al., "A modular and compact portable mini-endstation for highprecision, high-speed fixed target serial crystallography at FEL and synchrotron sources," Journal of Synchrotron Radiation, 22, 1372-1378 (2015).

[13] A. J. Bodey, Z. Z. Mileeva, T. Lowe et al., "Simultaneous heating and compression of irradiated graphite during synchrotron microtomographic imaging " Journal Of Physics: Conference Series X-Ray Microscopy Conference 2016 (XRM 2016). 849, (2017).

[14] E. Y. Guo, G. Zeng, D. Kazantsev et al., "Synchrotron X-ray tomographic quantification of microstructural evolution in ice cream - a multiphase soft solid," Rsc Advances, 7(25), 15561-15573 (2017).

[15] H. P. Menke, M. G. Andrew, J. Vila-Comamala et al., "Dynamic Pore-scale Reservoir-condition Imaging of Reaction in Carbonates Using Synchrotron Fast Tomography," Jove-Journal of Visualized Experiments(120), (2017).

[16] K. Singh, H. Menke, M. Andrew et al., "Dynamics of snap-off and pore-filling events during two-phase fluid flow in permeable media," Scientific Reports, 7, (2017).

[17] C. A. Reynolds, H. Menke, M. Andrew et al., "Dynamic fluid connectivity during steady-state multiphase flow in a sandstone," Proceedings of the National Academy of Sciences of the United States of America, 114(31), 8187-8192 (2017).

[18] D. S. Eastwood, P. M. Bayley, H. J. Chang et al., "Three-dimensional characterization of electrodeposited lithium microstructures using synchrotron X-ray phase contrast imaging," Chemical Communications, 51(2), 266-268 (2015).

[19] O. O. Taiwo, D. P. Finegan, J. M. Paz-Garcia et al., "Investigating the evolving microstructure of lithium metal electrodes in 3D using X-ray computed tomography," Physical Chemistry Chemical Physics, 19(33), 2211122120 (2017).

[20] A. J. Bodey, and C. Rau, "Launch of the I13-2 data beamline at the Diamond Light Source synchrotron ". 849. 